\title{
NEW CURRENT-MODE NOTCH AND ALLPASS FILTERS WITH SINGLE CURRENT DIFFERENCE AMPLIFIER
}

\author{
MUHAMMAD TAHER ABUELMA'ATTI \\ King Fahd University of Petroleum and Minerals, Box 203, Dhahran 31261, Saudi Arabia \\ (Received March 21, 1995; in final form May 5, 1995)
}

A new configuration for realization of current-mode notch and allpass filters is presented. It can synthesize second-order notch and allpass filters using a single current difference (Norton) amplifier and at most eight passive RC one port elements. Experimental results obtained from a notch filter realization are presented.

\section{INTRODUCTION}

At present, there is a growing interest in designing current-mode signal processing circuits. In these circuits, the current rather than the voltage is used as the active variable either throughout the whole circuit or only in certain critical areas. The use of current as the active parameter can result in circuits operating with higher signal bandwidths, greater linearity, and larger dynamic range than voltage-mode circuits [1]. The current conveyor, a powerful analog building block with current-mode capability, is therefore a strong potential candidate for implementing current-mode circuits, and recently a number of realizations have been presented using first- and second-generation current conveyors [see, for example, [2] and the references cited therein]. However, in many industrial electronic control systems, circuits are designed to operate off only a single power supply voltage. The current conveyor is typically designed for split power supplies and, therefore, current-mode active filters employing conventional current conveyors cannot be used in many industrial control applications. The current difference amplifier, designed to operate from a single power supply, is therefore, a strong candidate for such applications. A literature survey, [see, for example, [3]-[5] and the references cited therein] reveals that while the current-difference amplifier has been extensively used in designing voltage-mode biquadratic transfer functions, no attempt has been reported yet for its use in designing current-mode biquadratic filter circuits.

It is the purpose of this paper to present current-mode biquadratic notch and allpass filter realizations using the current-difference amplifier.

\section{PROPOSED CIRCUITS}

Consider the general circuit shown in Fig. 1. Assuming an ideal current difference amplifier defined by $i_{+}=i_{-}, v_{+}=v_{-} \cong 0$, routine analysis of the circuit of Fig. 1 


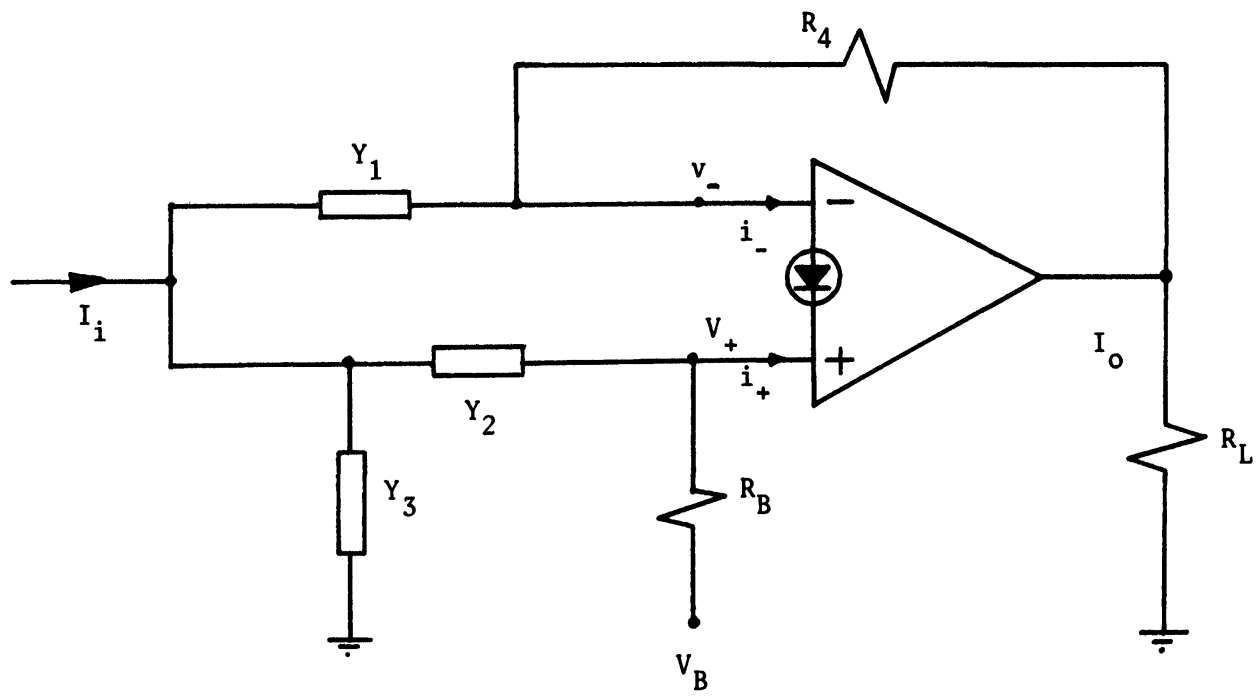

FIGURE 1 Proposed general circuit.

yields the current transfer function given by

$\frac{I_{o}}{I_{i}}=\frac{1}{G_{4} R_{L}} \frac{Y_{2}-Y_{1}}{Y_{1}+Y_{2}+Y_{3}}$

where $G_{4}=1 / R_{4}$.

Using (1), it is easy to show that current-mode notch and allpass filters can be realized. For example, if we choose, $\left.Y_{1}=1 /\left(R_{1}+1 / s C_{1}\right)\right), Y_{2}=1 / R_{2}+$ ${ }_{s} C_{2}, Y_{3}=0$ (open circuit), shown in Fig. 2(a), then (1) reduces to

$\frac{I_{o}}{I_{i}}=\frac{R_{4}}{R_{L}} \frac{1+s\left(C_{1} R_{1}+C_{2} R_{2}-C_{1} R_{2}\right)+s^{2} C_{1} C_{2} R_{1} R_{2}}{1+s\left(C_{1} R_{1}+C_{2} R_{2}+C_{1} R_{2}\right)+s^{2} C_{1} C_{2} R_{1} R_{2}}$

Now if

$C_{1} R_{1}+C_{2} R_{2}=C_{1} R_{2}$

then (2) reduces to

$$
H_{1}(s)=\frac{R_{4}}{R_{L}} \frac{s^{2}+\omega_{o}^{2}}{s^{2}+\frac{\omega_{o}}{Q_{o}} s+\omega_{o}^{2}}
$$

which corresponds to the transfer function of a second-order notch filter with the parameters

$\omega_{o}^{2}=\frac{1}{C_{1} C_{2} R_{1} R_{2}}$ 

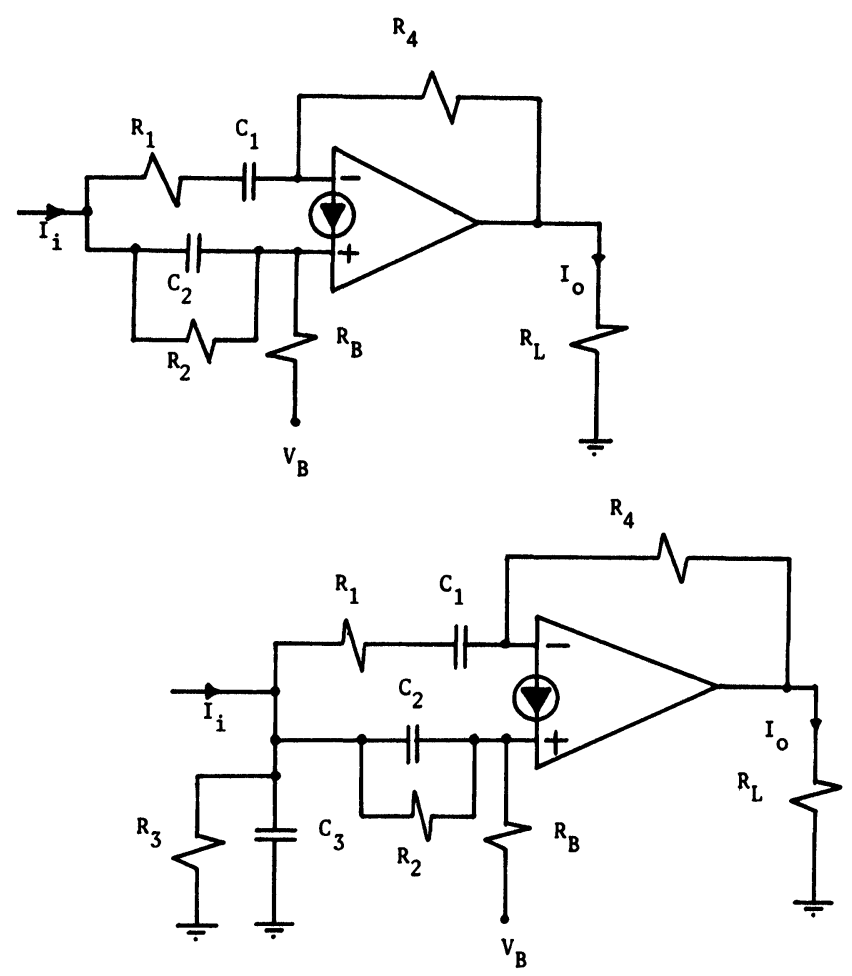

(a)

(b)

(c)

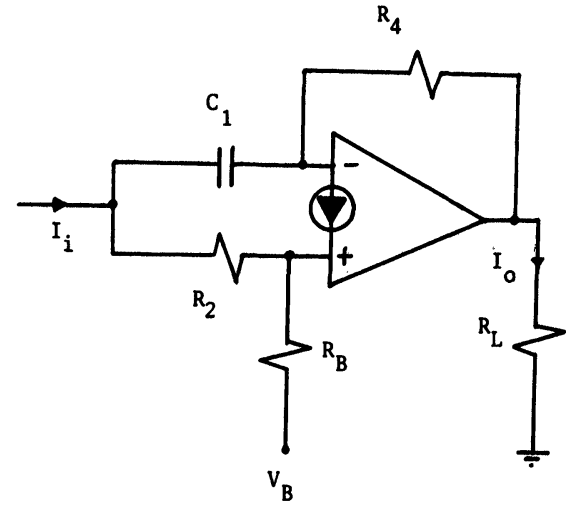

FIGURE 2 Proposed filter realizations (a) Second-order notch (b) Second-order allpass (c) First-order allpass.

and

$\frac{\omega_{o}}{Q_{o}}=\frac{1}{C_{1} R_{1}}+\frac{1}{C_{2} R_{2}}+\frac{1}{C_{2} R_{1}}$

Using (3), (5), and (6), the parameter $\frac{\omega_{o}}{Q_{o}}$ can be expressed by

$$
\frac{\omega_{o}}{Q_{o}}=\frac{2}{C_{2} R_{1}}
$$


Note that the ratio between the output and input currents of this notch filter is $R_{4} / R_{L}$. Such ratio can be made larger than one and, therefore, this notch realization is not suffering from a constant loss. Note also that this realization requires only six passive elements, including the load resistor $R_{L}$, plus one active element.

Now if a parallel RC combination is used for $Y_{3}$, that is $Y_{3}=1 / R_{3}+s C_{3}$ while $Y_{1}$ and $Y_{2}$ remain as before, shown in Fig. 2(b), then (1) reduces to

$\frac{I_{o}}{I_{i}}=\frac{R_{4}}{R_{L}} \frac{R_{3}}{R_{2}+R_{3}} \frac{1+s\left(C_{2} R_{2}+C_{1} R_{1}-C_{1} R_{2}\right)+s^{2} C_{1} C_{2} R_{1} R_{2}}{1+s\left(C_{1} R_{1}+\left(C_{1}+C_{2}+C_{3}\right) R_{p}\right)+s^{2}\left(C_{2}+C_{3}\right) C_{1} R_{1} R_{p}}=$

$\frac{R_{4}}{R_{L}} \frac{R_{3}}{R_{2}+R_{3}} \frac{1+\alpha s+\beta s^{2}}{1+\gamma s+\delta s^{2}}$

where $R_{p}+\frac{R_{2} R_{3}}{R_{2}+R_{3}}$

Now if $\alpha=-\gamma$, i.e.

$C_{2} R_{2}+\left(C_{1}+C_{2}+C_{3}\right) R_{p}+2 C_{1} R_{1}=C_{1} R_{2}$

and if $\beta=\delta$, i.e.

$C_{2} R_{2}=C_{3} R_{3}$

then $(8)$ reduces to

$H_{2}(s)=\frac{R_{4}}{R_{L}} \frac{R_{3}}{R_{2}+R_{3}} \frac{s^{2}-\frac{\omega_{o}}{Q_{o}} s+\omega_{o}^{2}}{s^{2}+\frac{\omega_{o}}{Q_{o}} s+\omega_{o}^{2}}$

which corresponds to the transfer function of a second-order allpass filter with the parameters

$\omega_{o}^{2}=\frac{1}{C_{1} C_{2} R_{1} R_{2}}$

and

$\frac{\omega_{o}}{Q_{o}}=\frac{C_{1} R_{1}+\left(C_{1}+C_{2}+C_{3}\right) R_{p}}{C_{1} C_{2} R_{1} R_{2}}$

Using (9), (12) and (13), the parameter $\frac{\omega_{o}}{Q_{0}}$ can be expressed by

$\frac{\omega_{o}}{Q_{o}}=\frac{1}{C_{2} R_{1}}-\frac{1}{C_{1} R_{1}}-\frac{1}{C_{2} R_{2}}$ 
Finally, if we choose $Y_{1}=1 / C_{1}, Y_{2}=1 / R_{2}$ while $Y_{3}=0$, shown in Fig. 2(c), then

$\frac{I_{o}}{I_{i}}=\frac{-R_{4}}{R_{L}} \frac{s C_{1} R_{2}-1}{s C_{1} R_{2}+1}$

Equation (15) corresponds to the transfer function of a first-order allpass filter. Note that the ratio between the output and input currents of this allpass filter is $R_{4} / R_{L}$. Such ratio can be made larger than one. Note also that this realization requires only four passive elements, including the load resistor, $R_{L}$, plus one active element.

\section{SENSITIVITY ANALYSIS}

By defining the sensitivity of a parameter $F$ to the element of variation $x_{i}$ by

$$
\mathrm{S}_{x i}^{F}=\frac{x_{i}}{F} \frac{d F}{d x_{i}}
$$

the passive sensitivities of the parameters $\omega_{o}$ and $\frac{\omega_{o}}{Q_{o}}$ of the proposed circuits of Fig. 2(a) and (b) have been calculated using equations (5), (7), (12), and (14) and their values are given in Table I. From Table I, one can easily see that all the passive $\omega_{o}$-sensitivities and $\frac{w_{o}}{Q_{o}}$-sensitivities of the second-order notch filters are $\leq 1$. One can also see that while the passive $\omega_{o}$-sensitivities of the second-order allpass filter are $\leq 1$, the $\frac{\omega_{o}}{Q_{o}}$-sensitivities may be appreciably high due to the presence of the

TABLE I

The passive sensitivities for the notch and allpass realizations of Figs. 2(a) and (b)

Function

Notch of Fig. 2(a)

Allpass of Fig. 2(b)
Passive Sensitivities

$$
S_{C_{1}}^{\omega_{o}}=S_{C_{2}}^{\omega_{o}}=S_{R_{1}}^{\omega_{o}}=S_{R_{2}}^{\omega_{o}}=-\frac{1}{2}
$$

$$
S_{R_{1}}^{\omega} d Q_{0}=S_{C_{2}}^{\omega} d Q_{0}=-\frac{1}{2}
$$

$$
S_{C_{1}}^{\omega_{o}}=S_{C_{2}}^{\omega_{o}}=S_{R_{1}}^{\omega_{o}}=S_{R_{2}}^{\omega_{o}}=-\frac{1}{2}
$$

$$
S_{R_{1}}^{\omega} d Q_{0}=\frac{-1}{1+\frac{R_{1}}{R_{2}} \frac{1}{C_{2} / C_{1}-1}}
$$$$
S_{R_{2}}^{\omega_{d} d Q_{o}}=\frac{-1}{1+\frac{R_{2}}{R_{1}}\left(\frac{C_{2}}{C_{1}}-1\right)}
$$

$$
S_{C_{1}}^{\omega_{d} Q_{o}}=\frac{-1}{1+\frac{C_{1}}{C_{2}}\left(\frac{R_{1}}{R_{2}}-1\right)}
$$

$$
S_{C_{2}}^{\omega_{d} / Q_{o}}=\frac{-1}{1+\frac{C_{2}}{C_{1}} \frac{1}{R_{1} / R_{2}-1}}
$$


difference terms in the denominators. However, the sensitivity figures may be made low, in the range of the notch filter realization, by careful design.

The effect of the finite gain of the current-difference amplifier on the performance of the proposed circuits can be studied by replacing $G_{4}$ in equation (1) by $G_{4}+\frac{G_{4}+Y_{1}}{A}$ where $A$ is the finite gain of the amplifier [4]. Thus, (1) reduces to $\frac{I_{o}}{I_{i}}=\frac{1}{\left(G_{4}+\frac{G_{4}+Y_{1}}{A}\right) R_{L}} \frac{Y_{2}-Y_{1}}{Y_{1}+Y_{2}+Y_{3}}$

For the second-order notch-filter realization with $Y_{1}=1 /\left(R_{1}+1 / s C_{1}\right), Y_{2}=$ $1 / R_{2}+s C_{2}, Y_{3}=0$, equation (16) reduces to

$$
\begin{aligned}
& \frac{I_{o}}{I_{i}}=\frac{R_{4}}{R_{L}(1+1 / A)} \frac{1+s C_{1} R_{1}}{1+s\left(C_{1} R_{1}+C_{1} R_{4} /(1+A)\right)} \\
& \frac{1+s\left(C_{1} R_{1}+C_{2} R_{2}-C_{1} R_{2}\right)+s^{2} C_{1} C_{2} R_{1} R_{2}}{1+s\left(C_{1} R_{1}+C_{2} R_{2}+C_{1} R_{2}\right)+s^{2} C_{1} C_{2} R_{1} R_{2}}
\end{aligned}
$$

The transfer function of (17) can be decomposed into two cascaded transfer functions $T_{1}(s)$ and $T_{2}(s)$. The first transfer function, $T_{1}(s)$, is expressed by

$$
T_{1}(s)=\frac{1+s C_{1} R_{1}}{1+s C_{1}\left(R_{1}+R_{4} /(1+A)\right)}
$$

This transfer function corresponds to a lowpass filter with high-frequency gain determined by the ratio $\frac{R_{4}}{R_{1}} \frac{1}{1+A}$. Both the pole $\omega_{p}=1 / C_{1}\left(R_{1}+R_{4} /(1+A)\right)$ and the zero $\omega_{z}=1 / C_{1} R_{1}$ in this transfer function are adjustable through $C_{1}$, but the ratio is held constant. The second transfer function, $T_{2}(s)$, is expressed by

$$
T_{2}(s)=\frac{R_{4}}{R_{L}(1+1 / A)} \frac{1+s\left(C_{1} R_{1}+C_{2} R_{2}-C_{1} R_{2}\right)+s^{2} C_{1} C_{2} R_{1} R_{2}}{1+s\left(C_{1} R_{1}+C_{2} R_{2}+C_{1} R_{2}\right)+s^{2} C_{1} C_{2} R_{1} R_{2}}
$$

This transfer function is the same as the transfer function of (2) with the gain slightly modified to $R_{4} / R_{L}(1+1 / A)$ rather than $R_{4} / R_{L}$. The notch filter parameters $\omega_{o}$ and $\omega_{o} / Q_{o}$ will remain the same inspite of the finite gain of the amplifier. Thus, by selecting $\frac{R_{4}}{1+1 / A} \ll R_{1}$ the notch characteristics of the circuit of Fig. 2(a) will preserve its shape.

In a similar way, if we take into consideration the effect of the finite gain of the amplifier, the transfer function of the allpass circuit of Fig. 2(b) will be

$$
\begin{gathered}
\frac{I_{o}}{I_{i}}=\frac{R_{4}}{(1+1 / A) R_{L}} \frac{R_{3}}{R_{2}+R_{3}} \frac{1+s C_{1} R_{1}}{1+s C_{1}\left(R_{1}+R_{4} /(1+A)\right)} \\
\frac{1+s\left(C_{2} R_{2}+C_{1} R_{1}-C_{1} R_{2}\right)+s^{2} C_{1} C_{2} R_{1} R_{2}}{1+s\left(C_{1} R_{1}+\left(C_{1}+C_{2}+C_{3}\right) R_{p}\right)+s^{2}\left(C_{2}+C_{3}\right) C_{1} R_{1} R_{p}}
\end{gathered}
$$


This transfer function can be decomposed into two cascaded transfer functions, $T_{1}(s)$, of equation (18), followed by the transfer function $T_{3}(s)$ given by

$T_{3}(s)=\frac{R_{4}}{(1+1 / A) R_{L}} \frac{R_{3}}{R_{2}+R_{3}} \frac{1+s\left(C_{2} R_{2}+C_{1} R_{1}-C_{1} R_{2}\right)+s^{2} C_{1} C_{2} R_{1} R_{2}}{1+s\left(C_{1} R_{1}+\left(C_{1}+C_{2}+C_{3}\right) R_{p}\right)+s^{2}\left(C_{2}+C_{3}\right) C_{1} R_{1} R_{p}}$

Equation (21) is the same as (8) with a slight modification in the gain due to the effect of finite gain of the amplifier. The allpass filter parameters $\omega_{o}$ and $\omega_{o} Q_{o}$ are not affected by the finite gain of the amplifier. Thus by selecting $R_{4} /(1+A)<<R_{1}$, the effect of the finite gain of the amplifier can be minimized, and the allpass characteristic of the circuit of Fig. 2(b) will preserve its shape.

\section{DESIGN PROCEDURE}

Design procedure is given for the notch and allpass filter circuits of Figs. 2(a) and (b). For the notch filter circuit of Fig. 2(a), the parameters $\omega_{o}^{2}$ and $\frac{\omega_{o}}{Q_{o}}$ are given by equations (5) and (7). Combining (5) and (7), then

$C_{1} R_{2}=\frac{1}{2 Q_{o} \omega_{o}}$

Thus, for given values of $\omega_{o}$ and $Q_{o}$, design values for $C_{1}, C_{2}, R_{1}$ and $R_{2}$ can be obtained that satisfy equations (3), (7), and (22) with $Q_{o} \leq 0.25$. For example, $R_{2}$ $=2 R_{1}=2 R, C_{1}=2 C_{2}=2 C$ gives $\omega_{o}=\frac{1}{2 C R}$ and $Q_{o}=0.25$. Values of $\mathrm{C}$ and $\mathrm{R}$ can be selected to yield the required $\omega_{o}$.

Similarly, for the allpass circuit of Fig. 2(b), the parameters $\omega^{2}{ }_{o}$ and $\frac{\omega_{o}}{Q_{o}}$ are given by equations (12) and (14). From (14), one can see that values of $C_{1}, C_{2}^{Q_{0}}, R_{1}$ and $R_{2}$ musty satisfy the condition

$\frac{1}{C_{2} R_{1}}>\frac{1}{C_{1} R_{1}}+\frac{1}{C_{2} R_{2}}$

Thus using (10), (12), (14), and (23), design values for $C_{1}, C_{2}, C_{3}, R_{1}, R_{2}$ and $R_{3}$ for given values of $\omega_{o}$ and $Q_{o}$ with $Q_{o} \leq 1$ are obtained. For example, $R_{2}=3 R_{1}=R_{3}$ $=3 R$ and $C_{1}=3 C_{2}=3 C_{3}=3 C$ gives $\omega_{o}=1 / 3 C R$ and $Q_{o}=1$.

\section{EXPERIMENTAL RESULTS}

The proposed notch and allpass filters were realized using the LM3900 currentdifference amplifier. In the laboratory, the input current $I_{i}$ was obtained using the Howland voltage-to-current converter [6]. The results obtained for the notch filter with $2 R_{1}=2 R_{4}=R_{2}=R_{B}=2 R_{L}=3.2 \mathrm{~K}, C_{1}=2 C_{2}=2 n F$ and $V_{B}=9 \mathrm{~V}$ are shown in Fig. 3. From Fig. 3, it is easy to see that the notch occurs at $49 \mathrm{KHz}$ while the predicted value using eqn (5) is $49.736 \mathrm{KHz}$. Thus, the experimental results are in good agreement with the theoretical analysis presented here. 


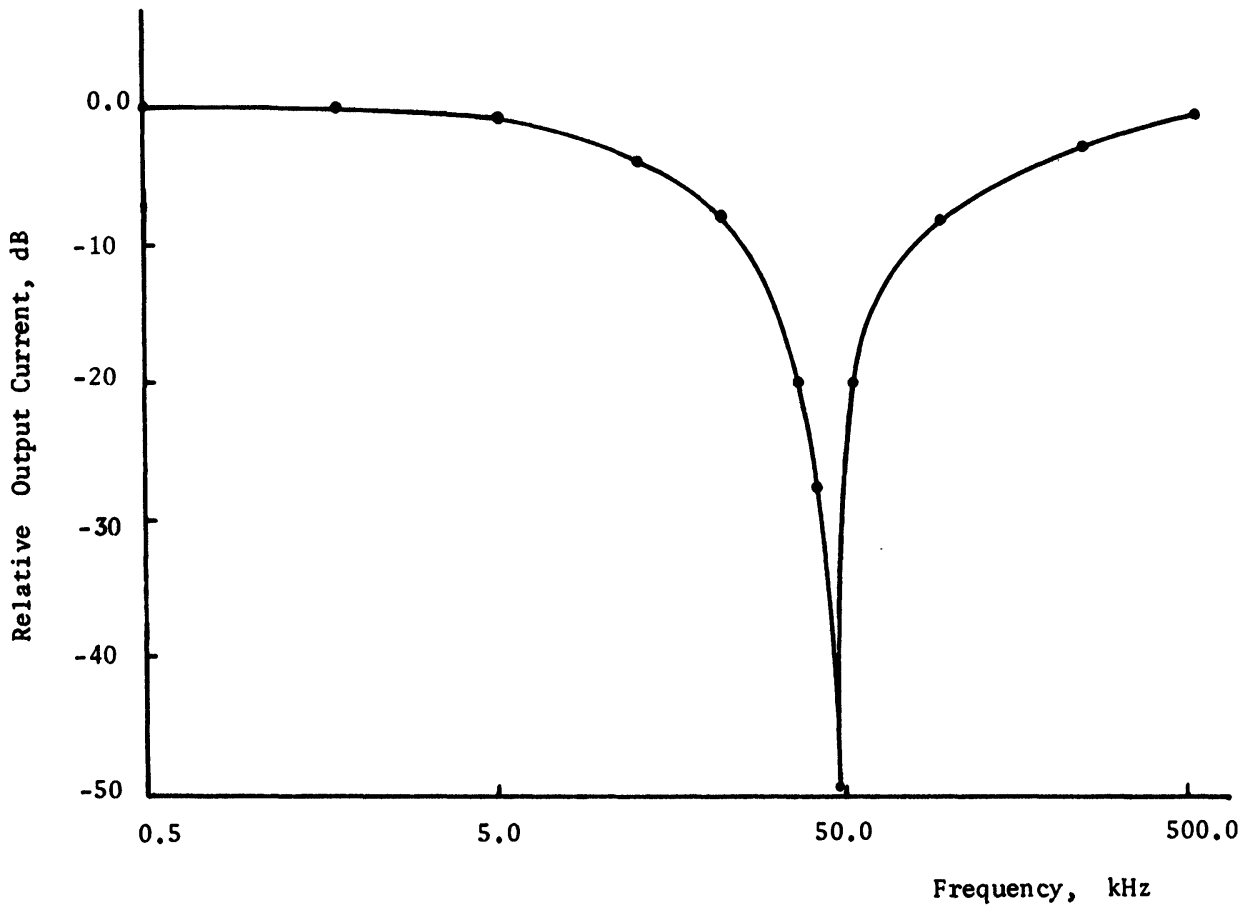

FIGURE 3 Measured notch filter characteristic using $2 R_{1}=2 R_{4}=R_{2}=R_{B}=2 R_{L}=3.2 \mathrm{k} \Omega, C_{1}=$ $2 C_{2}=2 n F_{9} V_{B}=9 V$.

\section{CONCLUSION}

A new circuit for realizing current-mode, second-order notch and allpass filters has been presented. The proposed circuit uses a single current difference (Norton) amplifier and at most eight passive RC one-port elements, including the load resistor. The circuit can also realize a first-order allpass filter using a single current difference amplifier and four passive elements only including the load resistor. The current-difference amplifier requires a single dc power supply and, therefore, the proposed realizations are very useful for many industrial electronic control systems designed to operate off only a single power supply voltage. The proposed realizations enjoy low active and passive sensitivities.

\section{REFERENCES}

1. B. Wilson, Recent developments in current conveyors and current-mode circuits, Proceedings IEE, Vol. 137, Part G, 1990, pp. 63-70

2. M.T. Abuelma'atti, New current-mode-active filters employing current conveyors, International Journal of Circuit Theory and Applications, Vol. 21, 1993, pp. 93-99

3. J.H. Brodie, Realization of nth order transfer functions using current differencing amplifiers, International Journal of Electronics, Vol. 44, 1978, pp. 663-665

4. J.H. Brodie, Realization of all-pass filter sections using current-differencing amplifiers, International Journal of Electronics, Vol. 53, 1982, pp. 319-330

5. T.M. Frederiksen, W.M. Howard and R.S. Sleeth, The LM3900-A new current-differencing quad of \pm input amplifiers, Linear Applications, National Semiconductors, pp. AN72, February 1973

6. S. Franco, Design with operational amplifiers and analog integrated circuits, McGraw-Hill; New York, 1988 

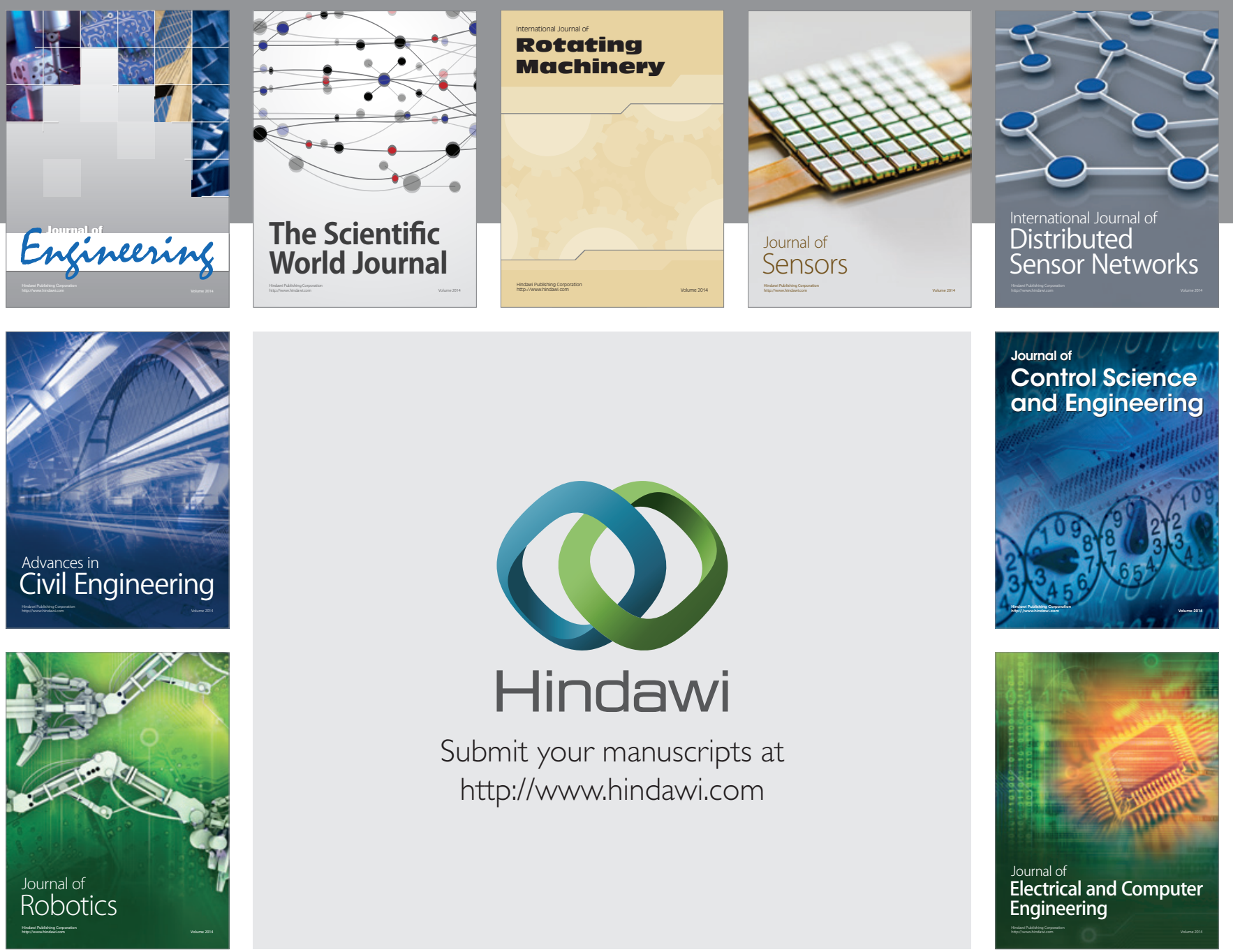

Submit your manuscripts at

http://www.hindawi.com
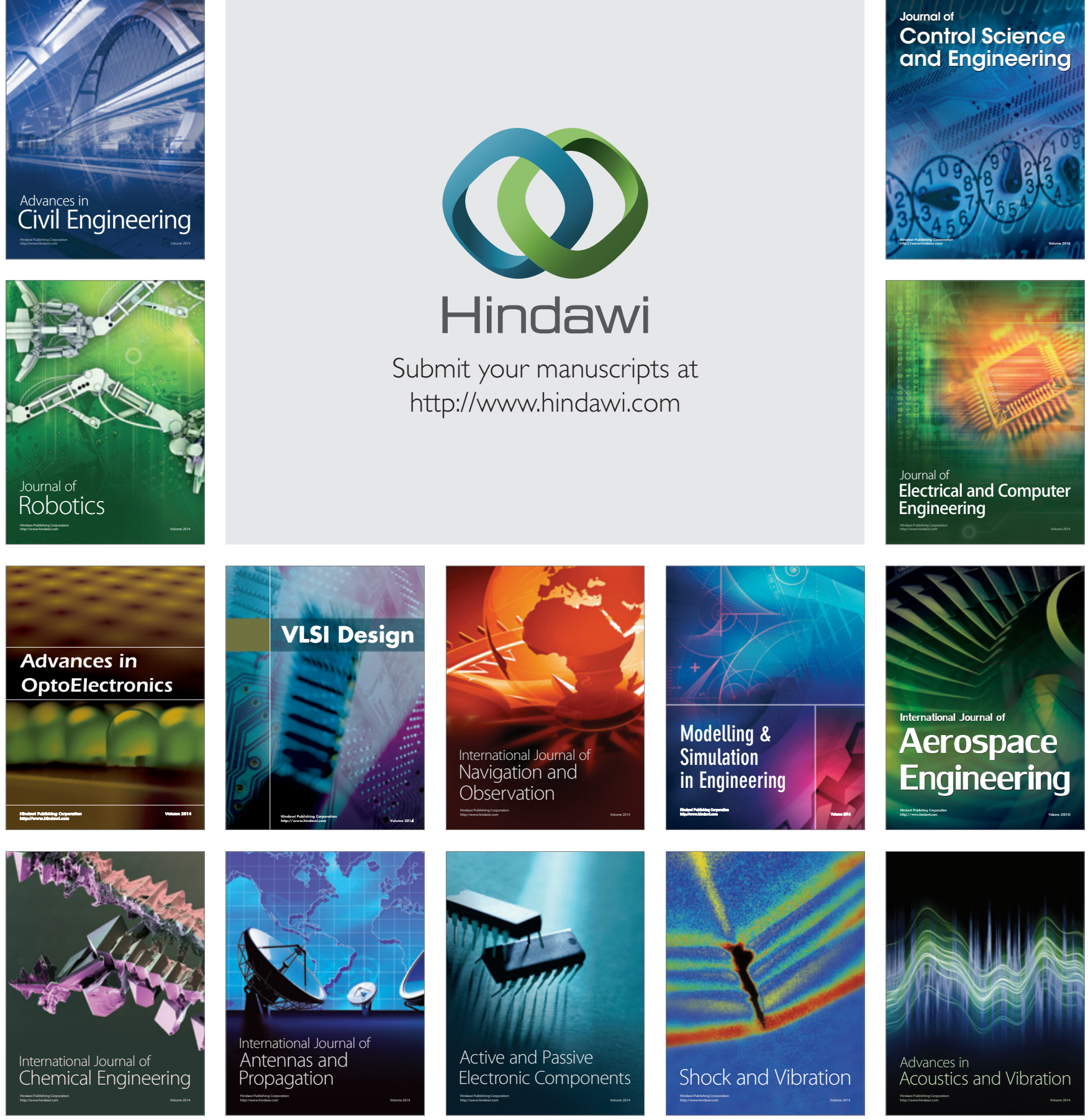